\title{
Obstetric and Neonatal Outcomes Related To Intrapartum Hydration Strategies in Low-Risk Deliveries: An Observational Study
}

Ana B. Hernández-López ( $\boldsymbol{Q}$ anabelen.hernandez2@salud.madrid.org )
Hospital Universitario Puerta de Hierro Majadahonda

\section{Cristina Muriel-Miguel}

Hospital Universitario Puerta de Hierro Majadahonda

\section{Tirso Pérez-Medina}

Hospital Universitario Puerta de Hierro Majadahonda

Aurora Fernández-Cañadas Morillo

Hospital Universitario Puerta de Hierro Majadahonda

\section{Carolina López-Lapeyrere}

Hospital Universitario Puerta de Hierro Majadahonda

Ángel Salcedo-Mariña

Hospital Universitario Puerta de Hierro Majadahonda

Inocencia Fornet-Ruiz

Hospital Universitario Puerta de Hierro Majadahonda

\section{Esther Rubio-González}

Hospital Universitario Puerta de Hierro Majadahonda

\section{Ana Royuela-Vicente}

Instituto de Investigación Sanitaria Puerta de Hierro-Segovia de Arana

\section{Montserrat Solís-Muñoz}

Hospital Universitario Puerta de Hierro Majadahonda

\section{Research Article}

Keywords: hydration labor, intrapartum care, midwife, newborn, obstetric results

Posted Date: August 13th, 2021

DOl: https://doi.org/10.21203/rs.3.rs-776979/v1

License: (1) This work is licensed under a Creative Commons Attribution 4.0 International License. Read Full License 


\section{Abstract \\ Background}

Effective myometrial contractility is important for successful labor, although little attention has been paid to the effect of managing intrapartum fluid intake. Ineffective myometrial contractility leads to prolonged labor, thus increasing obstetric and neonatal adverse outcomes. The risk of prolonged labor can be reduced by increasing the total volume of fluids administered during labor.

\section{Objective}

To determine the hydration strategies applied in nulliparous women undergoing low risk labor and their association with obstetric and neonatal outcomes.

\section{Methods}

A prospective cohort study was conducted in a Universitary Hospital. The study population included nulliparous women who presented in active labor or induced labor. Sample size was 147. In order to stratify women based on the hydration received, we set as a cut-off point the mean total volume administered per hour $(300 \mathrm{ml} / \mathrm{h})$. This enabled to compare obstetric, clinical, and neonatal outcomes in women who had received $\geq$ $300 \mathrm{~mL} / \mathrm{h} 0<300 \mathrm{~mL} / \mathrm{h}$. The primary outcome was total length of labor. Secondary outcomes included maternal and neonatal outcomes.

\section{Results}

The study population comprised 148 nulliparous women, mean (DS) age 32.2 (4.4) years, mean (DS) gestational age of 39.4 (1.41) weeks. At admission, median (IQR) dilation was $2(1-3) \mathrm{cm}$. Labor was induced in $65.5 \%(n=97)$. Obstetric and neonatal outcomes were more favorable in women who received $a \geq 300 \mathrm{~mL} / \mathrm{h}$ volume, with statistically significant median differences in the duration total duration of labor (526 vs $735 \mathrm{~min}$; $\mathrm{p}<0.001)$. Clinically relevant differences were also observed with respect to cesarean delivery $(14.3 \% \mathrm{vs}$ $18.7 \%)$, fever ( $5.5 \%$ vs $7.7 \%)$, weight loss at 24 hours $(-2.3 \%$ vs $-3 \%)$ and at 48 hours $(-5.7 \%$ vs $-6.3 \%)$, incidence of weight loss $>7 \%$ at 48 hours $(28.6 \%$ vs $39.8 \%)$, breastfeeding $(94.6 \%$ vs $82.4 \%)$.

\section{Conclusions}

Higher fluid volume administered to nulliparous women during low-risk labor is associated with improved obstetric and neonatal outcomes.

\section{Introduction}


Appropriate maternal hydration during pregnancy and labor is essential to ensure oxygenation of the fetus, supply of nutrients, and elimination of myometrial material [1, 2]. As a muscular organ, the uterus functions during labor and the puerperium and requires an adequate water supply in order to ensure efficient contractions [3]. Nevertheless, the impact of intrapartum fluid management on its functioning is unknown [4]. Inappropriate hydration during labor could lead to alterations in the acid-base balance in the fluid surrounding the fibers of the myometrium, thus leading to reduced contractility, prolonged labor [5], and increased probability of cesarean delivery [6]. Increased fluid intake has been shown to improve the performance of skeletal muscle during long periods of exercise $[7,8]$. Extrapolation of these data to uterine smooth muscle could explain the inadequate hydration affecting women who experience prolonged labor [9].

Prolonged labor is more common among nulliparous women and is associated with an increased risk of chorioamnionitis and hemorrhage; it is considered one of the main indications for nonscheduled cesarean delivery during the first and second stages of labor $[10,11]$. Intrapartum maternal fever complicates the process and may be associated with chorioamnionitis, which is usually treated with antibiotics [12]. However, a review of the literature suggests that this approach is not always suitable. The noninfectious causes of this condition, which are often overlooked, include the use of epidural anesthetic to relieve pain, changes in temperature during labor, premature rupture of membranes lasting > 24 hours, and use of oxytocin [12, 13].

Few studies have evaluated hydration in pregnant women. McKenzie et al. [14] observed that women with darker urine had higher urine osmolality values and therefore recommended increasing fluid intake to improve general hydration status. Oral fluid intake is the easiest method for replacing lost fluids. More than $500 \mathrm{~mL} / \mathrm{h}$ is thought to be necessary during prolonged physical exercise $[15,16]$. Mendelson suggested that aspiration in women who receive general anesthesia during labor could be prevented by stopping oral intake [17]. However, clear fluid intake during labor does not produce obstetric or neonatal adverse effects [18]. Some centers apply restrictive policies with respect to fluid intake, although such an approach contradicts the recommendations of the National Institute for Health and Care Excellence (NICE) [19] and the American Society of Anesthesiologists (ASA) [20].

Management of fluid volume and type of solution administered to women during labor varies and very often depends on the attending health professional. This variability can affect the woman's safety [21]. The metaanalysis of Dawood et al. [22] (9 trials, 1781 women) concluded that intravenous fluids reduced the duration of labor. The number of cesarean deliveries fell when women received intravenous solutions at $250 \mathrm{~mL} / \mathrm{h}$ compared with $125 \mathrm{~mL} / \mathrm{h}$. However, differences in the methodology and quality of some of the trials made it impossible to reach an acceptable level of evidence to recommend specific hydration strategies (oral and intravenous).

Chantry et al. [23] reported an association between weight loss in the newborn and the volume of fluid administered to the mother during the first stage of labor. Weight loss in the newborn is considered excessive when greater than $7 \%-10 \%$ during the first 48 hours of life [24-28].

The objective of the present study was to determine hydration strategies applied in nulliparous women with low-risk labor and their role in obstetric and neonatal outcomes. The data provided are aimed at reducing variability in clinical practice in such a way that the volume of solution (total volume administered orally and 
intravenously during labor and puerperium) and the type of solution administered can be adjusted to the woman's needs during labor, without the risk of associated complications.

\section{Methods}

\section{Study design and participants}

We performed a prospective cohort study in the delivery room of Hospital NAME (City, Country), which manages more than 3000 births per year. The study participants comprised nulliparous women with a singleton pregnancy aged $\geq 18$ years and their newborns, who were attended for normal births by midwives in the Obstetrics Department of a University Hospital.

The inclusion criteria were as follows: (a) term pregnancy (week $37_{0}-41_{3}$ ), (b) cephalic presentation, (c) spontaneous active labor ( $3 \mathrm{~cm}$ in cervical examination with regular uterine contractions), membranes ruptured/not ruptured, (d) or induced labor (1 cm in cervical examination, with no regular contractions), for which the main clinical indications were premature rupture of membranes or post-term pregnancy $\left(>41_{3}\right.$ weeks of amenorrhea). The exclusion criteria were language barrier, multiple pregnancy, gestational diabetes, preeclampsia, heart disease, kidney disease, fever on admission, and precipitate labor.

\section{Sample size}

Given that the potentially eligible study population was 1300 women, with an expected standard deviation for a total duration of labor of 4.98 hours (pilot study) and a $99 \%$ confidence interval and $1 \%$ precision, the resulting sample size was 147 . The analysis was performed with Epidat version 4.2. Sampling was non probabilistic and based on order of arrival at the delivery room.

\section{Study Procedures}

The midwife of the obstetric emergency department verified the inclusion criteria of the woman on admission to the delivery room. The woman was provided with information and invited to participate in the study after providing her written informed consent. During labor and the immediate puerperium ( 2 hours after delivery), the attending midwife completed the case report form. After this time, mother and baby were taken to a room on the Obstetrics Ward, where they remained until discharge (48-72 hours postpartum) and were followed up by the attending nurse. The study coordinator was responsible for verifying the quality of data collection on the case report form throughout the process of caring for the mother and the newborn.

\section{Study measurements and data collection}

\section{Maternal variables}

- Main Outcomes: total duration of labor (from admission to delivery).

- Secondary Outcomes: duration of the first stage of labor (from admission to the delivery room until complete dilation), duration of the second stage of labor (from full dilation to delivery), prolonged labor (duration of labor > 12 hours) [1], interval between administration of epidural analgesia and delivery, duration of oxytocin, cesarean delivery, and intrapartum fever (temperature $\geq 38^{\circ} \mathrm{C}$ ) $[29,30]$. 
- Other obstetric outcomes: Type of delivery, dry mouth (VAS), thirst (VAS), biochemical parameters (sodium, potassium, creatinine, and osmolality in blood and urine) at admission and during puerperium, diuresis $(\mathrm{mL} / \mathrm{h})$, urine color (Armstrong scale) [31]. Thirst, dry mouth, and pain were evaluated considering the VAS score $(0-10)$.

- Socio-demographic variables: age, race/ethnic group.

- Clinical variables on admittance, intrapartum, and puerperium (2 hours after birth): gestational age, number of pregnancies, type of labor, reason for induction, rupture of membranes, dry mouth, presence of beta-hemolytic streptococcus, cervical examination (dilation in $\mathrm{cm}$, Bishop test), arterial blood pressure, heart rate, temperature, pain (visual analog scale [VAS]), temperature in the delivery room, total fluid volume administered (oral and intravenous) during labor and the immediate puerperium, amount of oxytocin (mU), type of analgesia, time with ruptured membranes, diuresis, blood loss, active management of the third stage (administration of $5 \mathrm{IU}$ of intravenous oxytocin following delivery of the anterior shoulder) [32, 33].

\section{Neonate variables}

- Main Outcomes: Percentage weight loss at 24 and 48 hours, complications, breastfeeding.

- Newborn variables: sex, weight (at birth, 24 hours, and 48 hours), biochemical parameters in umbilical cord blood (sodium, potassium, and glucose), initiation of breastfeeding, type of feeding at 48 hours, jaundice (24 and 48 hours), respiratory distress [34], transplacental hyponatremia [35] (serum sodium [cord] $<135 \mathrm{meq} / \mathrm{l}$ ), hypoglucemia [36] (serum glucose [cord] $<40 \mathrm{mg} / \mathrm{dL}$ ), admission to the neonatal intensive care unit.

\section{Analysis}

A descriptive analysis of the categorical variables was performed based on absolute and relative frequencies. Numerical variables were analyzed using the mean and standard deviation (SD) and the median and interquartile range (IQR) depending on the normality of their distribution. The univariate analysis was performed using the Mann-Whitney test to compare numerical variables and the chi-squared test or Fisher exact test to compare categorical variables. Statistical significance was set at $p<0.05$. The analysis was performed using version Stata 16.0 .

\section{Results}

The study population comprised 148 nulliparous women with a mean (SD) age of 32.2 (4.4) years and a mean (SD) gestational age of 39.4 (1.41) weeks. At admission, median (IQR) dilation was $2(1-3) \mathrm{cm}$. Labor was induced in $65.5 \%(n=97)$ of cases. The characteristics of the study sample can be seen in Table 1. 
Table 1

Characteristics of the study sample

\begin{tabular}{|c|c|}
\hline \multicolumn{2}{|l|}{ VARIABLES. $N=148$ STATISTICS } \\
\hline Age, mean (SD) & $32.2(4.4)$ \\
\hline Gestational age in weeks, mean (SD) & $39.4(1.4)$ \\
\hline \multicolumn{2}{|l|}{ Race/Ethnic group, n (\%) } \\
\hline White & $136(92.0)$ \\
\hline Latina & $10(7.0)$ \\
\hline Other & $2(1.0)$ \\
\hline \multicolumn{2}{|l|}{ Number of pregnancies, $\mathrm{n}(\%)$} \\
\hline None & $122(82.4)$ \\
\hline Multiple & $26(17.6)$ \\
\hline \multicolumn{2}{|l|}{ Labor, n (\%) } \\
\hline Spontaneous & $51(34.5)$ \\
\hline Induced & $97(65.5)$ \\
\hline Spontaneous rupture of membranes & $53(54.6)$ \\
\hline Post-term pregnancy (>41 weeks +3 days) & $24(24.8)$ \\
\hline Maternal disease & $5(5.2)$ \\
\hline Fetal disease & $8(8.2)$ \\
\hline Other (oligohydramnios, polyhydramnios, other) & $7(7.2)$ \\
\hline Pain (VAS) $(n=147)$, mean (SD) & $4.2(3.2)$ \\
\hline$<3$ (mild), n (\%) & $62(42.2)$ \\
\hline 4-7 (moderate), n (\%) & $63(42.8)$ \\
\hline$\geq 8$ (severe), n (\%) & $22(15.0)$ \\
\hline Dry mouth at admission $(n=145), n(\%)$ & $86(59.3)$ \\
\hline Beta-hemolytic streptococcus positive, $n$ (\%) & $21(14.2)$ \\
\hline \multicolumn{2}{|l|}{ Cervical examination } \\
\hline Bishop test, median (IQR) & $5.0(3.0-8.0)$ \\
\hline Spontaneous labor $(n=51)$ & $8.0(7.0-9.0)$ \\
\hline Induced labor $(n=97)$ & $4.0(2.5-6.0)$ \\
\hline
\end{tabular}

Abbreviations: SD, standard deviation; IQR, interquartile range; VAS, Visual Analog Scale. 


\begin{tabular}{|ll|}
\hline VARIABLES. $\mathbf{N}=\mathbf{1 4 8}$ STATISTICS & \\
\hline Dilation $(\mathbf{c m})$, median (IQR) & $2.0(1.0-3.0)$ \\
\hline Spontaneous labor $(\mathrm{n}=51)$ & $3.0(3.0-4.0)$ \\
\hline Induced labor $(\mathrm{n}=97)$ & $1.0(1.0-2.0)$ \\
\hline Temperature, median (IQR) & $36.2(36.0-36.6)$ \\
\hline Systolic blood pressure, median (IQR) & $121.0(114.0-128.7)$ \\
\hline Diastolic blood pressure, median (IQR) & $76.0(70.0-82.7)$ \\
\hline Heart rate, median (IQR) & $84.5(75.0-93.7)$ \\
\hline Abbreviations: SD, standard deviation; IQR, interquartile range; VAS, Visual Analog Scale. \\
\hline
\end{tabular}

Epidural anesthesia was administered in $96.5 \%(n=137)$ of cases. During labor, the median (IQR) dose was $225(100,6-408,6) \mathrm{mU} / \mathrm{h}$ for oxytocin, $215.4(171.3-265.5) \mathrm{mL} / \mathrm{h}$ for intravenous serum (mainly saline solution and Ringer lactate solution), and $62.6(37.6-92.4) \mathrm{mL} / \mathrm{h}$ for oral fluids (mainly water and isotonic drinks). The mean (SD) total volume administered per hour was 301.9 (160.9) $\mathrm{mL} / \mathrm{h}$. Mean (SD) diuresis per hour was 73.5 (42.9) $\mathrm{mL} / \mathrm{h}$ (Table 2). 
Table 2

Obstetric management and total volume administered during labor and puerperium.

\begin{tabular}{|ll|}
\hline LABOR. $\mathrm{N}=148$ & Median (IQR) \\
\hline Fluid $\mathrm{mL} / \mathrm{h}(\mathrm{n}=146)$ & $215.4(171.3-265.5)$ \\
\hline Saline $(\mathrm{mL})(\mathrm{n}=142)$ & $1200.0(1000.0-1600.0)$ \\
\hline Ringer lactate $(\mathrm{mL})(\mathrm{n}=137)$ & $1000.0(1000.0-1500.0)$ \\
\hline Dextrose saline $5 \%(\mathrm{~mL})(\mathrm{n}=11)$ & $500.0(500.0-500.0)$ \\
\hline Voluven $(\mathrm{mL})(\mathrm{n}=6)$ & $500.0(400.0-1025.0)$ \\
\hline Oral intake $\mathrm{mL} / \mathrm{h}(\mathrm{n}=145)$ & $62.6(37.6-92.4)$ \\
\hline Total fluid volume $\mathrm{mL} / \mathrm{h}(\mathrm{n}=147)$ & $287.9(232.1-339.9)$ \\
\hline Diuresis $\mathrm{mL} / \mathrm{h}(\mathrm{n}=145)$ & $64.9(42.4-87.7)$ \\
\hline Oxytocin $\mathrm{mU} / \mathrm{h}(\mathrm{n}=130)$ & $225(100,6-408,6)$ \\
\hline Active management of the third stage, $\mathrm{n}(\%)$ & $116(78.4)$ \\
\hline Estimated blood loss $(\mathrm{mL})(\mathrm{n}=58)$ & $184(147.7-247.7)$ \\
\hline Epidural anesthesia $(\mathrm{n}=142), \mathrm{n}(\%)$ & $137(96.5)$ \\
\hline Room temperature $(\mathrm{n}=139)$ & $25(25-26)$ \\
\hline PUERPERIUM. $\mathrm{N}=148$ & \\
\hline Fluid $\mathrm{mL} / \mathrm{h}(\mathrm{n}=109)$ & $250(0.0-500.0)$ \\
\hline Saline $(\mathrm{mL})(\mathrm{n}=101)$ & $0(0-300)$ \\
\hline Ringer lactate $(\mathrm{mL})(\mathrm{n}=100)$ & $0(0-100)$ \\
\hline Oral intake $\mathrm{mL} / \mathrm{h}(\mathrm{n}=113)$ & $0.0(0.0-0.0)$ \\
\hline Total fluid volume $\mathrm{mL} / \mathrm{h}(\mathrm{n}=108)$ & $59.0(10.0-250.0)$ \\
\hline Diuresis, $\mathrm{mL} / \mathrm{h}(\mathrm{n}=114)$ & $25(25-26)$ \\
\hline Oxytocin $\mathrm{mU} / \mathrm{h}(\mathrm{n}=112)$ & \\
\hline Room temperature $(\mathrm{n}=109)$ & $(90.0-275.0)$ \\
\hline Abbreviation: $\mathrm{IQR}, \mathrm{interquartile} \mathrm{range.}$ & \\
\hline
\end{tabular}

Delivery was spontaneous vaginal in $52 \%(n=77)$ of women. The median (IQR) duration of labor was 667 (493-848) minutes. Prolonged labor was recorded in $2.7 \%(n=3)$. Fever was recorded during labor in $6.9 \%(n=$ $10)$, with a lower incidence during the puerperium $(3.7 \% ; n=5)$. Urine biochemical parameters at admission had changed significantly in the puerperium ( $p<0.001)$, as follows: creatinine median (IQR) 87.3 [50.9-136.4] vs 48.3 [26.4-67.5] mg/dL, and osmolality median (IQR) 560.0 [400.5-759.5] vs 293.0 [202.0-427.0] (Table 3). 
Table 3

Obstetric and clinical outcomes in labor and puerperium

\section{VARIABLES STATISTICS VARIABLES STATISTICS}

LABOR. $\mathrm{N}=148$

Regular contractions, $\mathrm{n}(\%)$

Mild diaphoresis, $\mathrm{n}(\%)$

First stage (min), median (IQR)

Second stage (min), median (IQR)

Total time (min), median (IQR)

Time with ruptured membranes $(\mathrm{n}=$ 144), median (IQR)
PUERPERIUM. $\mathbf{N}=148$

$108(73)$

$136(92)$

535.0

$(369.8-$

750.8)

$110.0(0.0-$

320)

$667(493-$

848)

534.5

(327.3-

874.8)

$446.0(303-$

613.5)

525.0

(374.5-

789)

Type of delivery, $n(\%)$

Spontaneous vaginal

$77(52.0)$

Instrumental

$46(31.1)$

Cesarean

$25(16.9)$

Uterine atony, $\mathrm{n}(\%)$

$1(0.6)$

Prolonged labor $>12 h(n=111), n(\%) \quad 3(2.7)$

Temperature $(n=147)$, median (IQR)

36.4

(36.1-

Temperature $(n=135)$, median

(IQR)

$37.2(36.7-$ 36.7)

37.5)

$119(80.9)$

$<37.7^{\circ} \mathrm{C}, \mathrm{n}(\%)$

$121(89.6)$

$<37.7^{\circ} \mathrm{C}, \mathrm{n}(\%)$

18 (12.2)

$37.7-37.9^{\circ} \mathrm{C}, \mathrm{n}(\%)$

9 (6.7)

$\geq 38^{\circ} \mathrm{C}, \mathrm{n}(\%)$

$10(6.9)$

$\geq 38^{\circ} \mathrm{C}, \mathrm{n}(\%)$

5 (3.7)

Systolic blood pressure, median (IQR)

119 (110-

127)

Systolic blood pressure $(n=$

118

(109.5-

125)

Abbreviations: SD, standard deviation; IQR, interquartile range; VAS, Visual Analog Scale. 


\section{VARIABLES STATISTICS VARIABLES STATISTICS}

\begin{tabular}{|c|c|c|c|}
\hline Diastolic blood pressure, median (IQR) & $70(66-79)$ & $\begin{array}{l}\text { Diastolic blood pressure }(n= \\
\text { 140), median (IQR) }\end{array}$ & $77(60-72)$ \\
\hline Heart rate, median (IQR) & $\begin{array}{l}78(71- \\
87.5)\end{array}$ & $\begin{array}{l}\text { Heart rate }(n=139) \text {, median } \\
(\text { IQR })\end{array}$ & $78(68-88)$ \\
\hline Dry mouth, n (\%) & $96(64.9)$ & Dry mouth $(\mathrm{n}=117), \mathrm{n}(\%)$ & $91(77.8)$ \\
\hline Thirst (VAS), mean (SD) & $3.9(2.1)$ & $\begin{array}{l}\text { Thirst (VAS) }(n=109) \text {, mean } \\
(S D)\end{array}$ & $6.3(2.9)$ \\
\hline Pain (VAS) $(n=145)$, mean (SD) & $3.2(2.2)$ & Pain (VAS) $(n=125)$, mean (SD) & $1.9(2.4)$ \\
\hline $\begin{array}{l}\text { Choluria (Armstrong) }(n=143) \text {, mean } \\
(S D)\end{array}$ & $4.5(1.1)$ & $\begin{array}{l}\text { Choluria (Armstrong) }(\mathrm{n}=112) \\
\text { mean (SD) }\end{array}$ & $3.94(1.61)$ \\
\hline Laboratory values at admission & & $\begin{array}{l}\text { Laboratory values } 2 \text { hours after } \\
\text { delivery }\end{array}$ & \\
\hline $\begin{array}{l}\text { Sodium (blood) mmol/L (135-145) }(\mathrm{n}= \\
\text { 144), median (IQR) }\end{array}$ & $\begin{array}{l}137.0 \\
(135.0- \\
138.0)\end{array}$ & $\begin{array}{l}\text { Sodium (blood) } \mathrm{mmol} / \mathrm{L}(\mathrm{n}= \\
\text { 108), median (IQR) }\end{array}$ & $\begin{array}{l}138.0 \\
(135.3- \\
139.0)\end{array}$ \\
\hline $\begin{array}{l}\text { Potassium (blood) mmol/L (3.5-5.0) (n } \\
=139) \text {, median (IQR) }\end{array}$ & $\begin{array}{l}4.1(3.9- \\
4.3)\end{array}$ & $\begin{array}{l}\text { Potassium (blood) } \mathrm{mmol} / \mathrm{L}(\mathrm{n}= \\
\text { 104), median (IQR) }\end{array}$ & $\begin{array}{l}3.8(3.7- \\
4.1)\end{array}$ \\
\hline $\begin{array}{l}\text { Creatinine (blood) } \mathrm{mg} / \mathrm{dL}(0.5-0.9)(\mathrm{n}= \\
94) \text {, median (IQR) }\end{array}$ & $\begin{array}{l}0.5(0.4- \\
0.6)\end{array}$ & $\begin{array}{l}\text { Creatinine (blood) } \mathrm{mg} / \mathrm{dL}(\mathrm{n}= \\
\text { 74), median (IQR) }\end{array}$ & $\begin{array}{l}0.5(0.4- \\
0.6)\end{array}$ \\
\hline $\begin{array}{l}\text { Osmolality (blood) mOsm/kg (275- } \\
\text { 305) }(n=136) \text {, median (IQR) }\end{array}$ & $\begin{array}{l}283.0 \\
(279.0- \\
287.0)\end{array}$ & $\begin{array}{l}\text { Osmolality (blood) mOsm/kg ( } \\
=108) \text {, median (IQR) }\end{array}$ & $\begin{array}{l}281.0 \\
(276.0- \\
285.0)\end{array}$ \\
\hline $\begin{array}{l}\text { Sodium (urine) mmol/L }(25-145)(n= \\
\text { 143), median (IQR) }\end{array}$ & $\begin{array}{l}99.0(72.0- \\
134.0)\end{array}$ & $\begin{array}{l}\text { Sodium (urine) } \mathrm{mmol} / \mathrm{L}(\mathrm{n}= \\
\text { 108), median (IQR) }\end{array}$ & $\begin{array}{l}66.0(39.0- \\
107.2)\end{array}$ \\
\hline $\begin{array}{l}\text { Potassium (urine) } \mathrm{mmol} / \mathrm{L}(15-100) \text { ( } \\
=143) \text {, median (IQR) }\end{array}$ & $\begin{array}{l}51.0 \\
(32.2- \\
84.0)\end{array}$ & $\begin{array}{l}\text { Potassium (urine) } \mathrm{mmol} / \mathrm{L}(\mathrm{n}= \\
\text { 108), median (IQR) }\end{array}$ & $\begin{array}{l}30.4(16.9- \\
47.9)\end{array}$ \\
\hline $\begin{array}{l}\text { Creatinine (urine) } \mathrm{mg} / \mathrm{dL}(60-200)(\mathrm{n}= \\
\text { 99), median (IQR) }\end{array}$ & $\begin{array}{l}87.3(50.9- \\
136.4)\end{array}$ & $\begin{array}{l}\text { Creatinine (urine) } \mathrm{mg} / \mathrm{dL}(\mathrm{n}= \\
\text { 74), median (IQR) }\end{array}$ & $\begin{array}{l}48.3(26.4- \\
67.5)\end{array}$ \\
\hline $\begin{array}{l}\text { Osmolality (urine) } \mathrm{mOsm} / \mathrm{kg}(\mathrm{n}=101) \text {, } \\
\text { median (IQR) }\end{array}$ & $\begin{array}{l}560.0 \\
(400.5- \\
759.5)\end{array}$ & $\begin{array}{l}\text { Osmolality (urine) mOsm/kg ( } \mathrm{n} \\
=75 \text { ), median (IQR) }\end{array}$ & $\begin{array}{l}293.0 \\
(202.0- \\
427.0)\end{array}$ \\
\hline
\end{tabular}

Abbreviations: SD, standard deviation; IQR, interquartile range; VAS, Visual Analog Scale.

The $53.4 \%(n=79)$ of the newborns were girls. Breastfeeding was started in the first few hours in $87.2 \%(n=$ 129). Weight loss $>7 \%$ at 48 hours was recorded in $34.4 \%(n=51)$ of the newborns. Four of the 148 newborns had to be admitted to the Neonatal Intensive Care Unit: respiratory distress $(n=2)$, phototherapy in newborn with low weight $(n=1)$, capillary glycemia $<40 \mathrm{mg} / \mathrm{dL}$ associated with low weight $(n=1)$ (Table 4). 
Table 4

Characteristics of newborns and neonatal outcomes

\begin{tabular}{|c|c|}
\hline \multicolumn{2}{|l|}{ VARIABLES. $N$ = 148 Median (IQR) } \\
\hline Female, n (\%) & $79(53.4)$ \\
\hline Birth weight (g) & $3186(2891-3490.5)$ \\
\hline Sodium (cord) $\mathrm{mmol} / \mathrm{L}(135-145)(n=134)$ & $135.0(134-137)$ \\
\hline Potassium (cord) mmol/L $(3.0-5.0)(n=118)$ & $5.4(4.9-6.2)$ \\
\hline Glucose (cord) mg/dL $(60-100)(n=131)$ & $71.0(61-77)$ \\
\hline Breastfeeding, $\mathrm{n}(\%)$ & $129(87.2)$ \\
\hline \multicolumn{2}{|l|}{ AT 24 HOURS } \\
\hline Weight (g) & $3080.0(2806.5-3378.5)$ \\
\hline Weight loss (\%) & $-2.8(-4.2,-1.5)$ \\
\hline \multicolumn{2}{|l|}{ AT 48 HOURS } \\
\hline Weight (g) & $2964.0(2718.0-3240.0)$ \\
\hline Weight loss (\%) & $-6.1(-7.5,-4.7)$ \\
\hline Weight loss $>7 \%, n(\%)$ & $51(34.4)$ \\
\hline Icterus, $\mathrm{n}(\%)$ & $35(23.6)$ \\
\hline Breastfeeding, $n(\%)$ & $129(87.2)$ \\
\hline \multicolumn{2}{|l|}{ COMPLICATIONS } \\
\hline Admission to the Neonatal Intensive Care Unit, $\mathrm{n}(\%)$ & $4(2.7)$ \\
\hline Respiratory distress, $\mathrm{n}(\%)$ & $2(1.3)$ \\
\hline Phototherapy, n (\%) & $1(0.7)$ \\
\hline Capillary glycemia < 40 mg/dL, n (\%) & $1(0.7)$ \\
\hline
\end{tabular}

In order to stratify women based on the hydration received, we set as a cut-off point the mean total volume administered per hour ( $300 \mathrm{~mL} / \mathrm{h}$ ). This enabled us to compare obstetric, clinical, and neonatal outcomes in women who had received $\geq 300 \mathrm{~mL} / \mathrm{h}$ with those who had received $<300 \mathrm{~mL} / \mathrm{h}$. Obstetric and clinical outcomes were more favorable in women who received a higher volume than in those who received a lower volume (Table 5), with statistically significant median differences in the duration of the first stage of labor (420 vs 585 min; $p=0.009$ ), total duration of labor ( 526 vs $735 \mathrm{~min} ; p<0.001$ ), duration of analgesia (403 vs $480 ; p$ $=0.019$ ), and duration of oxytocin ( 466 vs $592 ; p=0.044)$. Clinically relevant differences were also observed with respect to cesarean delivery ( $14.3 \%$ vs $18.7 \%$ ) and fever ( $5.5 \%$ vs $7.7 \%)$. No women spent more than 24 
hours with ruptured membranes: maximum time was 22.94 hours in the group that received $<300 \mathrm{~mL} / \mathrm{h}$ and 22.43 hours in the group that received $\geq 300 \mathrm{~mL} / \mathrm{h}$. 
Table 5

Obstetric and neonatal outcomes according to volume administered during labor

\begin{tabular}{|c|c|c|c|}
\hline \multirow[t]{2}{*}{ VARIABLES } & $\begin{array}{l}<300 \mathrm{~mL} / \mathrm{h}(\mathrm{n}= \\
91)\end{array}$ & 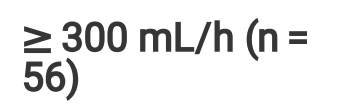 & $\begin{array}{l}p \\
\text { values }\end{array}$ \\
\hline & Median (min-max) & \multicolumn{2}{|l|}{ Median (min-max) } \\
\hline \multicolumn{4}{|l|}{ OBSTETRIC AND CLINICAL OUTCOMES } \\
\hline First stage of labor (min) & $\begin{array}{l}585.0(90.0- \\
1310.0)\end{array}$ & $\begin{array}{l}420.0(60.0- \\
1770.0)\end{array}$ & 0.009 \\
\hline Second stage of labor (min) & $115.0(0.0-320.0)$ & $101.5(0.0-240.0)$ & 0.323 \\
\hline Total duration of labor (min) & $\begin{array}{l}735.0(122.0- \\
1309.0)\end{array}$ & $\begin{array}{l}526.0(126.0- \\
1665.0)\end{array}$ & $<0.001$ \\
\hline Time with ruptured membranes $(\mathrm{n}=144)$ & $572.5(3-1374)$ & $479.5(52-1346)$ & 0.234 \\
\hline Duration of analgesia $(n=141)$ & $480(6-1087)$ & $403.0(58-1047)$ & 0.019 \\
\hline Duration of oxytocin $(n=141)$ & $592.0(3-1279)$ & $466.0(5-1377)$ & 0.044 \\
\hline Instrumental delivery, n (\%) & $28(30.8)$ & $17(30.3)$ & 0.686 \\
\hline Cesarean delivery, n (\%) & $17(18.7)$ & $8(14.3)$ & 0.686 \\
\hline Temperature $\geq 38^{\circ} \mathrm{C}(\mathrm{n}=146), \mathrm{n}(\%)$ & $7(7.7)$ & $3(5.5)$ & 0.474 \\
\hline Dry mouth during labor, $\mathrm{n}(\%)$ & $62(67)$ & $34(60.7)$ & 0.273 \\
\hline Dry mouth during puerperium, $\mathrm{n}(\%)$ & $55(37)$ & $37(25)$ & 0.745 \\
\hline Thirst during labor (VAS) $(n=146)$, mean (SD) & $3.9(2.1)$ & $3.8(2.1)$ & 0.722 \\
\hline $\begin{array}{l}\text { Thirst during puerperium (VAS) }(n=108) \text {, mean } \\
\text { (SD) }\end{array}$ & $6.4(3.1)$ & $6.2(2.7)$ & 0.662 \\
\hline Oxytocin mU/h ( $n=138)$, median (IQR) & $4800(1948-8420)$ & $4260(2032-7888)$ & 0.589 \\
\hline Diuresis $\mathrm{mL} / \mathrm{h}(\mathrm{n}=145)$ & $64.5(41.5-82.9)$ & $73.4(48.3-112.6)$ & 0.082 \\
\hline Choluria during labor $(\mathrm{n}=142)$, mean (SD) & $4.5(1.1)$ & $4.4(1.1)$ & 0.674 \\
\hline Choluria during puerperium $(\mathrm{n}=111)$, mean $(S D)$ & $3.9(1.6)$ & $3.9(1.6)$ & 0.907 \\
\hline \multicolumn{4}{|l|}{$\begin{array}{l}\text { LABORATORY VALUES AT } 2 \text { HOURS AFTER } \\
\text { DELIVERY }\end{array}$} \\
\hline Sodium (blood) mmol/L (135-145) $(n=108)$ & $\begin{array}{l}137.5(126.0- \\
141.0)\end{array}$ & $\begin{array}{l}138.0(126.0- \\
142.0)\end{array}$ & 0.386 \\
\hline Potassium (blood) mmol/L) $(3.5-5.0)(n=104)$ & $3.9(3.4-5.3)$ & $3.8(3.3-5.4)$ & 0.775 \\
\hline Creatinine (blood) mg/dL (0.5-0.9) $(\mathrm{n}=74)$ & $0.5(0.2-1.2)$ & $0.5(0.2-0.9)$ & 0.355 \\
\hline Osmolality (blood) mOsm/kg $(275-305)(n=108)$ & $\begin{array}{l}281.5(249.0- \\
302.0)\end{array}$ & $\begin{array}{l}281.0(271.0- \\
300.0)\end{array}$ & 0.677 \\
\hline
\end{tabular}

Abbreviations: SD, standard deviation; IQR, interquartile range; VAS, Visual Analog Scale. 


\section{VARIABLES}

$<300 \mathrm{~mL} / \mathrm{h}(\mathrm{n}=$

91)

Median (min-max) $\geq 300 \mathrm{~mL} / \mathrm{h}(\mathrm{n}=$

56)

$p$

values

Median (min-max)
Sodium (urine) meq/L $(25-145)(n=108)$

Potassium (urine) meq/L $(15-100)(n=108)$

Creatinine (urine) $\mathrm{mg} / \mathrm{dL}(60-200)(n=74)$

Osmolality (urine) $\mathrm{mOsm} / \mathrm{kg}(\mathrm{n}=75)$
$69.5(11.0-245.0)$

$28.8(10.2-136.4)$

$44.3(9.1-145.0)$

$291.0(52.0-874.0)$
$62.0(10.0-208.0)$

$31.9(10.0-275.0)$

$48.3(10.6-125.7)$

$287.5(146.0-$ 815.0)
0.602

0.764

0.600

0.863

NEONATAL OUTCOME

Birth

Weight (g), median (IQR)

$3242(2960-3480)$

$3062(2878-3500)$

0.302

Sodium (cord) $\mathrm{mmol} / \mathrm{L}(135-145)(n=134)$

$135.0(125.0-$

141.0)

$135.0(130.0-$

0.974 143.0)

Potassium (cord) $\mathrm{mmol} / \mathrm{L}(3.0-5.0)(n=118)$

$5.3(4.8-6.0)$

$5.5(5.2-6.3)$

0.751

Glucose (cord) mg/dL (60-100) $(n=131)$

$70.0(25.0-110.0)$

$71.0(26.0-101.0)$

0.727

24 hours

Weight loss

$96.0(0.0-286.0)$

$77.5(0.0-268.0)$

0.170

Weight loss (\%), median (IQR)

$-3.04(-4.54 ;-$

$1.42)$

$-2.3(-3.3 ;-1.5)$

0.145

48 hours

Weight loss

$210.0(00.0-$

1270.0)

Weight loss (\%), median (IQR)

Weight loss $>7 \%, n(\%)$

$-6.3(-7.8 ;-4.7)$

35 (39.8)

$-5.71(-7.2 ;-4.7)$

0.305

Jaundice $(n=143), n(\%)$

$23(26.4)$

$16(28.6)$

0.171

Breastfeeding ( $n=147), n(\%)$

75 (82.4)

$12(21.4)$

0.497

$53(94.6)$

0.183

Complications, $\mathrm{n}(\%)$

Admission to intensive care

$3(2.0)$

$1(0.7)$

Abbreviations: SD, standard deviation; IQR, interquartile range; VAS, Visual Analog Scale. 


\begin{tabular}{|c|c|c|c|}
\hline \multirow[t]{2}{*}{ VARIABLES } & $\begin{array}{l}<300 \mathrm{~mL} / \mathrm{h}(\mathrm{n}= \\
91)\end{array}$ & $\begin{array}{l}\geq 300 \mathrm{~mL} / \mathrm{h}(\mathrm{n}= \\
\mathrm{5}=\end{array}$ & $\begin{array}{l}p \\
\text { values }\end{array}$ \\
\hline & Median (min-max) & Median (min-max) & \\
\hline Respiratory distress & $1(0.6)$ & $1(0.7)$ & \\
\hline Phototherapy & $1(0.7)$ & & \\
\hline Capillary glycemia $<40 \mathrm{mg} / \mathrm{dL}$ & $1(0.7)$ & & \\
\hline
\end{tabular}

The newborns were also affected by the greater or lesser volume per hour administered to the mothers (Table 5). Neonatal outcomes were more favorable in newborns whose mothers received a greater volume $(\geq 300$ $\mathrm{mL} / \mathrm{h})$. Lower values were recorded in children of the more hydrated mothers for percentage of weight loss at 24 hours $(-2.3 \%$ vs $-3 \% ; p=0.145)$ and 48 hours $(-5.7 \%$ vs $-6.3 \% ; p=0.305)$ and the incidence of weight loss $>7 \%$ at 48 hours $(28.6 \%$ vs $39.8 \% ; p=0.171)$. Breastfeeding was more frequent in the group that received a greater volume $(94.6 \%$ vs $82.4 \% ; p=0.183)$. Furthermore, three of four newborns who required admission to the neonatal intensive care unit belonged to the group of mothers who had received a lower volume.

\section{Discussion}

The findings suggest that obstetric and clinical outcomes were better in nulliparous women who received a total fluid volume of $\geq 300 \mathrm{~mL} / \mathrm{h}$ during labor than in those who received $\leq 300 \mathrm{~mL} / \mathrm{h}$. The lower values for duration of the first stage of labor, total duration of labor, duration of oxytocin, and duration of epidural anesthesia were statistically significant. Similarly, the incidence of prolonged labor (> 12 hours) was lower. Clinically relevant differences included a lower incidence of intrapartum fever and cesarean delivery. No woman spent more than 24 hours with ruptured membranes.

As for the newborns, more favorable, clinically relevant outcomes were recorded for those whose mothers received a total volume $\geq 300 \mathrm{~mL} / \mathrm{h}$. The most noteworthy findings were lower weight loss at 24 and 48 hours, lower incidence of weight loss $>7 \%$ at 48 hours, and greater incidence of breastfeeding. Furthermore, three of four newborns admitted to the neonatal ICU were the children of mothers who received a lower quantity of fluids $(<300 \mathrm{~mL} / \mathrm{h})$.

The findings associated with the duration of the first and second stages of labor and, total duration of labor are relevant, considering that the participants in the present study were recruited with a median cervical dilation of $2 \mathrm{~cm}$ (latent phase) compared with $4 \mathrm{~cm}$ (active phase) in the studies of Fong et al. [37] and Zhang et al. [38]. The median duration of the first stage of the better hydrated women ( $\geq 300 \mathrm{~mL} / \mathrm{h}$ ) was 420 minutes, which is superior to the results reported by Fong et al. [37] (399.5 minutes for the group receiving $2.5 \%$ dextrose in normal saline $[250 \mathrm{~mL} / \mathrm{h}])$, considering baseline dilation status. This results are consistent with those of Harper et al. [39].

As for the volume administered during labor, was observed a statistically significant association between total volume administered $\geq 300 \mathrm{~mL} / \mathrm{h}$ and reduced median duration of the first stage (420 min), total duration of 
labor (526 min), duration of analgesia (403 min), and duration of oxytocin (466 min). In this sense, previous studies found an association between the increase in total volume administered and reduced duration of labor $[21,22]$. One clinical trial [21] found that intravenous hydration significantly reduced the duration of active labor, the frequency of prolonged labor, and the administration of oxytocin in nulliparous women. A metaanalysis [22] showed that intravenous administration of fluids reduced the duration of labor.

The number of cesarean deliveries was greater in the lesser hydrated group $(<300 \mathrm{~mL} / \mathrm{h})$, with an incidence of $18.7 \%$ compared with $14.3 \%$ in the better hydrated group $(\geq 300 \mathrm{~mL} / \mathrm{h}$ ). The clinical trial (with 195 nulliparous women) by Garite et al. [1] (195 nulliparous women) analyzed the association between increased fluids during labor and progress of labor: the number of cesarean deliveries decreased when women received intravenous solutions at $250 \mathrm{~mL} / \mathrm{h}$ compared with $125 \mathrm{~mL} / \mathrm{h}^{1}$. Other studies were unable to demonstrate a similar effect $[18,22,37]$.

The incidence of intrapartum fever in the better hydrated group $(\geq 300 \mathrm{~mL} / \mathrm{h})$ was $5.5 \%$, which is more favorable than the data reported by Towers et al. [40] (7\%) and Braun et al. [41] (9\%) and like the percentage reported by Alexander et al. [42] (5\%).

The women in the present study had a median choluria value (Armstrong) of 4 in the puerperium, with median osmolality in urine of $<300 \mathrm{mOsm} / \mathrm{kg}$. A clinical trial performed in $2015^{14}$ and a descriptive study in 2013 [43] found that pregnant women with choluria $\geq 4$ probably had a urine osmolality of $\geq 500 \mathrm{mOsm} / \mathrm{kg}$. Therefore, the authors considered that increased fluid consumption could improve general hydration. The findings of the current study enable us to state that the women were generally well hydrated during labor, although optimal needs should be addressed in greater depth.

The percentage of weight loss at 24 and 48 hours and the incidence of weight loss $>7 \%$ at 48 hours was lower in the children of the better hydrated mothers $(\geq 300 \mathrm{~mL} / \mathrm{h}$ ). In this sense, some studies reported significant positive associations between the intrapartum fluid dose and weight loss [23, 24], whereas others did not report significant associations $[25,28]$.

With respect to cord blood biochemical parameters, the median (IQR) concentrations were as follows: sodium, 135 (134-137) mmol/L; potassium $5.4(4.9-6.2) \mathrm{mmol} / \mathrm{L}$; and glucose, $71(61-77) \mathrm{mg} / \mathrm{dL}$. These values are consistent with international recommendations $[44,45]$, and the ranges reported are like those found by Kratz et al. [46] and slightly higher than those reported by Bequer et al. [47].

\section{Clinical implications}

Hydration regimens improved during labor, with an increase in the volumes administered and a reduction in glucose solutions compared with crystalloid solutions. This decision was taken with the aim of reducing the risk of hypoglycemia in the neonate. Another modification, which was made at the initiation of the study, concerned antibiotic therapy. Antibiotics were administered initially to women whose temperature was $\geq 38^{\circ} \mathrm{C}$, although we found that temperature decreased in women who were better hydrated. Thus, in the case of fever, the first step was to increase the volume administered; antibiotics were only administered when the woman's temperature did not decrease.

\section{Research implications}


To consolidate the results of the cohort study, we proposed a randomized controlled clinical trial that would enable us to continue the research line initiated. The study protocol was published in 2020 with the title Efficacy of "optimal hydration" during labor: HYDRATA study protocol for a randomized clinical trial [48]. The trial was registered at www.clinicaltrials.gov, with number XXXXXXXXXXX.

\section{Strengths and limitations}

The study design is considered a strong point. The prospective cohort study allows obtaining relevant data on the association between hydration and obstetric and neonatal outcomes. The methodological rigor allowed the control of possible biases.

One limitation was the lack of some data, because they were not collected in routine practice, as was the case with laboratory values. Further analyzes revealed that women whose labor was induced may require a higher volume of fluids per hour to achieve optimal hydration. This hypothesis will be considered in the ongoing clinical trial.

\section{Conclusions}

A larger fluid volume ( $\geq 300 \mathrm{~mL} / \mathrm{h}$ ) administered to nulliparous women during labor was associated with improved obstetric outcomes (reduced duration of first stage of labor, total duration of labor, duration of oxytocin, duration of epidural anesthesia, and incidence of intrapartum fever and cesarean delivery) and improved neonatal outcomes (reduced weight loss at 24 and 48 hours, lower incidence of weight loss $>7 \%$ at 48 hours, greater incidence of breastfeeding, lower incidence of complications).

The findings of this study provide new evidence in favor of appropriate hydration for women during labor, which could improve the health and safety of mothers and their newborns and reduce maternal and fetal morbidity.

\section{Abbreviations}

\section{DS}

standard deviation

ICU

intensive care unit

IQR

interquartile range

VAS

visual analog scale

Vs

versus

\section{Declarations}

\section{Ethics approval and consent to participate}


The study was carried out in accordance with the Helsinki declaration, in compliance with Biomedical Research Law, and in accordance with Good Clinical Practice. All participants were volunteers who provided written informed consent. Patient confidentiality was guaranteed. The study was approved by the hospital's Clinical Research Ethics Committee.

\section{Consent for publication}

Not applicable.

\section{Availability of data and materials}

The data that support the findings of this study are not publicly available. Data are however available from the authors upon reasonable request and with permission of the hospital's Clinical Research Ethics Committee.

\section{Competing interests}

The authors declare that they have no conflicts of interest.

\section{Funding}

This study was funded by Fundación para la Investigación Biomédica del Hospital Universitario Puerta de Hierro Majadahonda (Madrid, Spain) for the period 2013-2015, after a peer-reviewed competitive process.

\section{Authors' contributions}

Ana B HERNÁNDEZ-LÓPEZ: Conceptualization, Methodology, Investigation, Resources, Writing (original draft), Supervision, Project administration, Acquisition of funding. Cristina MURIEL-MIGUEL: Investigation, Writing (review and editing). Tirso PÉREZ-MEDINA: Methodology, Writing (original draft). Aurora FERNANDEZCAÑADAS-MORILLO: Investigation, Writing (review and editing). Carolina LÓPEZ- LAPEYRERE: Investigation, Writing (review and editing). Ángel SALCEDO-MARIÑAS: Methodology, Writing (review and editing). Inocencia FORNET-RUIZ: Methodology, Writing (review and editing). Esther RUBIO-GONZÁLEZ: Methodology, Writing (review and editing). Ana ROYUELA-VICENTE: Formal analysis, Writing (review and editing). Montserrat SOLÍSMUÑOZ: Conceptualization, Methodology, Formal analysis, Writing (original draft), Acquisition of funding.

\section{Acknowledgements}

We are grateful to the women, their newborns, and their partners for their participation in the study. We acknowledge the work and dedication of all the professionals who participated in data collection.

\section{References}

1. Garite TJ, Weeks J, Peters-Phair K, et al. A randomized controlled trial of the effect of increased intravenous hydration on the course of labor in nulliparous women. Am J Obstet Gynecol.2000;183: 1544-1548.

2. EFSA Panel on Dietetic Products, Nutrition, and Allergies (NDA). Scientific Opinion on Dietary reference values for water. EFSA Journal.2010; 8(3):1459. 
3. Eslamian L, Marsoosi V, Pakneeyat Y. Increased intravenous fluid intake and the course of labor in nulliparous women. Int J Gynecol Obstet.2000; 93(2):102-105.

4. Toohill J, Soong B, Flenady V. Interventions for ketosis during labour. Cochrane Database Syst Rev.2008; 3:CD004230.

5. Pierce S J, Kupittayanant S, Shmygo T, Wray S. The effects of pH change on calcium signalling and force in pregnant human myometrium. Am J Obstet Gynecol.2003;188(4):1031-1038.

6. Greiss FC Jr, Anderson SG, Still JG. Relaciones de flujo de presión uterina durante la gestación temprana. Am J Obstet Gynecol.1976;126:799-808.

7. Dilts PV Jr, Brinkman CR 3d. Interrelaciones hemodinámicas uterinas y sistémicas y su respuesta a la hipoxia. Am J Obstet Gynecol.1969;103:138-44.

8. Harger-Domitrovich SG, McClaughry AE, Gaskill SE, Ruby BC. Exogenous carbohydrate spares muscle glycogen in men and women during 10h of exercise. Med Sci Sports Exerc.2007;39:2171-2179.

9. Hawley JA, Tipton KD, Millard-Stafford ML. Promoting training adaptations through nutritional interventions. J Sports Sci.2006;24:709-712.

10. Broach J, Newton N. Food and Beverages in Labor. Part I: Cross-Cultural and Historical Practices. BIRTH.1988;15:2.

11. Florica M, Stephansson O, Nordstrom L. Indications associated with increased cesarean section rates in a Swedish hospital. Int J Gynaecol Obstet.2006; 92:181-185.

12. Apantaku O, Mulik V. Maternal intra-partum fever. J Obstet Gynaecol.2007;27(1):12-15.

13. Lieberman E, Cohen A, Lang J, et al. Maternal intra-partum temperature elevation as a risk factor for caesarean delivery and assisted vaginal delivery. AJPH.1999; 89:506-510.

14. McKenzie AL, Muñoz CX, Ellis LA, et al. Urine color as an indicator of urine concentration in pregnant and lactating women. Eur J Nutr.2017; 56(1):355-62.

15. Cheng YW, Delaney SS, Hopkins LM, Caughey AB. The association between the length of first stage of labor, mode of delivery, and perinatal outcomes in women undergoing induction of labor. Am J Obstet Gynecol.2009; 201:477

16. Osborne C, Ecker JL, Gauvreau K, et al. Maternal temperature elevation and Occiput Posterior position at birth among Low-risk women receiving epidural analgesia. J Midwifery Womens Health.2011;56(5):446451.

17. Sperling JD, Dahlke JD, Sibai BM. Restriction of oral intake during labor: whither are we bound? AJOG.2016;214 (5): 592-596.

18. Kavitha A, Chacko KP, Thomas E, et al. A randomized controlled trial to study the effect of IV hydration on the duration of labor in nulliparous women. Arch Gynecol Obstet.2012; 285(2):343-346.

19. National Institute for Health and Care Excellence [NICE]. Intrapartum Care for healthy women and babies. Clinical Guideline (CG 190).2014. Accessed February 1, 2020. https://www.nice.org.uk/guidance/cg190.

20. American Society of Anesthesiologists Task Force on Obstetric Anesthesia (ASA). Practice guidelines for obstetric anesthesia: An updated report by the American Society of Anesthesiologists Task Force on Obstetric Anesthesia and the Society for Obstetric Anesthesia and Perinatology. Anesthesiology.2016;106(4):843-863. 
21. Direkvand-Moghadam A, Rezaeian M. Increased intravenous hydration of nulliparas in labor. Int $\mathrm{J}$ Gynaecol Obstet.2012;118(3): 213-215.

22. Dawood F, Dowswell T, Quenby S. Intravenous fluids for reducing the duration of labour in low-risk nulliparous women. Cochrane Database of Systematic Reviews.2013; Issue 6. Art. No.: CD007715.

23. Chantry CJ, Nommsen-Rivers LA, Peerson JM, et al. Excess weight loss in first-born breastfed newborns relates to maternal intrapartum fluid balance. Pediatrics.2011;127:171-179.

24. Noel-Weiss J, Woodend AK, Peterson WE, et al. An observational study of associations among maternal fluids during parturition, neonatal output, and breastfed newborn weight loss. Int Breastfeed J.2011;6(1):9.

25. Sheehan K, Faught BE, Plyley M, et al. The role of Intrapartum intravenous therapy and Newborn Weight loss: challenging the 7\% Rule. Clinics in Mother and Child Health.2015;20(2):1-4.

26. Lamp JM, Macke JK. Relationships among intrapartum maternal fluid intake, birth type, neonatal output, and neonatal weight loss during the first 48 hours after birth. J Obstet Gynecol Neonatal Nurs.2010; 39:169-177.

27. Thulier DJ. Maternal hydration and infant weight: a case-control study in breastfeeding infants delivered by cesarean section. Open Access Dissertations.2013; Paper 118. Accessed February 1, 2020. https://digitalcommons.uri.edu/oa_diss/118.

28. Eltonsy S, Blinn A, Sonier B, et al. Intrapartum intravenous fluids for caesarean delivery and newborn weight loss: a retrospective cohort study. BMJ Paediatrics Open.2017; 1: e000070.

29. Ferrero S, López M, Cobo T, Palacio M. Protocolo: Fiebre Intraparto. Fiebre Puerperal. Centre de Medicina Fetal i Neonatal de Barcelona;2016. Accessed February 1, 2020. https://medicinafetalbarcelona.org/protocolos/es/patologia-materna-obstetrica/fiebre\%20puerperal.pdf.

30. Acker DB, Schulman EB, Ransil BJ, et al. The normal parturient's admission temperature. Am J Obstet Gynecol.1987;157:308.

31. Armstrong LE, Maresh CM, Castellani JW, et al. Urinary indices of hydration status. Int J Sport Nutr.1994;4:265-279.

32. SEGO. Hemorragia posparto precoz. Prog Obstet Ginecol.2008;51(8):497-505.

33. Grupo de trabajo de la Guía de Práctica Clínica sobre la atención al parto normal. Guía de Práctica Clínica sobre la atención al parto normal. Plan de Calidad para el Sistema Nacional de Salud del Ministerio de Sanidad y Política Social. Guías de Práctica Clínica en el SNS: OSTEBA Nº 2009/01; 2010. Accessed February 1, 2020.

https://www.mscbs.gob.es/organizacion/sns/planCalidadSNS/pdf/equidad/guiaPracticaClinicaParto.pdf

34. Coto GD, López J, Fernández B, et al. Recién nacido a término con dificultad respiratoria: enfoque diagnóstico y terapéutico. Asociación Española de Pediatría (AEP). 2008;30:285-305. Accessed February 1, 2020. https://www.aeped.es/sites/default/files/documentos/30.pdf.

35. Marcialis MA, Dessi A, Pintus MC, et al. Neonatal hyponatremia: differential diagnosis and treatment. J Matern Fetal Neonatal Med.2011;24 Suppl 1:75-79.

36. Fernández JR, Couce M, Fraga JM. Hipoglucemia neonatal. Asociación Española de Pediatría (AEP). 2008;18:159-168. Accessed February 1, 2020.

https://www.aeped.es/sites/default/files/documentos/18_1.pdf

Page 20/21 
37. Fong A, Serra AE, Caballero D, et al. A randomized, double-blinded, controlled trial of the effects of fluid rate and/or presence of dextrose in intravenous fluids on the labor course of nulliparas. Am J Obstet Gynecol.2017;208 - 17.e1-7.

38. Zhang J, Landy HJ, Branch DW, Reddy UM. Contemporary patterns of spontaneous labor with normal neonatal outcomes. Obstet Gynecol.2010;116:1281-1287.

39. Harper LM, Caughey AB, Odibo AO, et al. Normal progress of induced labor. Obstet Gynecol.2012; 119:1113-1118.

40. Towers CV, Yates A, Zite N, et al. Incidence of fever in labor and risk of neonatal sepsis. Am J Obstet Gynecol.2017; 216:596. e1-5.

41. Braun $D$, Bromberger $P, H o N J$, Getahun D. Low rate of perinatal sepsis in term infants of mothers with chorioamnionitis. Am J Perinatol. 2016;32:143-150.

42. Alexander JM, McIntire DM, Leveno KJ. Chorioamnionitis and the prognosis for term infants. Obstet Gynecol.1999; 94:274-278.

43. Malisova O, Protopappas A, Nyktari A, et al. Estimations of water balance after validating and administering the water balance questionnaire in pregnant women. Int J Food Sci Nutr.2014;65(3):280285.

44. Ehsanipoor R M, Saccoce G, Seligman, N S, et al. Intravenous fluid rate for reduction of cesarean delivery rate in nulliparous women: A systematic review and meta-analysis. Acta Obstet Gynecol Scand.2017;96(7):804-811.

45. Schanler RJ, Lynn C, Abrams S. Nutrición parenteral y enteral. In: Ballard T, editor. Tratado de neonatología de Avery. 7th. ed. Madrid: Elsevier; 2010:944-961.

46. Kratz A, Ferraro M, Sluss PM. Massachusetts General Hospital: laboratory values. N Engl J Med.2004; 351(15):1549-1563.

47. Bequer $L$, Gómez T, Pérez de Alejo $L$, et al. Perfil bioquímico y valores de referencia en sangre del cordón umbilical. ABCL.2014;48(3):311-317.

48. AUTORS. Article title. Journal.2020;xx(x):x-xx. 